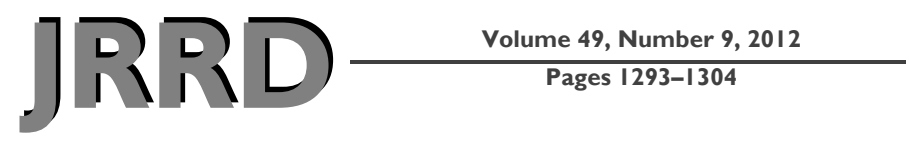

\title{
Quantifiable patterns of limb loading and unloading during hemiparetic gait: Relation to kinetic and kinematic parameters
}

\author{
Bhavana Raja, PT, PhD; ${ }^{1}$ Richard R. Neptune, PhD; ${ }^{2}$ Steven A. Kautz, PhD $^{3^{*}}$ \\ ${ }^{1}$ Rehabilitation Science Doctoral Program, University of Florida, Gainesville, FL; ${ }^{2}$ Department of Mechanical Engi- \\ neering, The University of Texas, Austin, TX; ${ }^{3}$ Ralph H. Johnson Department of Veterans Affairs Medical Center, \\ Charleston, SC; and Department of Health Sciences and Research and Division of Physical Therapy, College of Health \\ Professions, Medical University of South Carolina, Charleston, SC
}

\begin{abstract}
Persons with poststroke hemiparesis are characterized by asymmetry in limb loading (LL) and limb unloading (LU), which has been reported in static and quasi-static tasks but has not been quantified during walking. The purpose of this study was to determine the asymmetry in magnitude and duration of LL and LU in individuals with hemiparesis and its relationship with functional walking status and specific kinematic and kinetic variables during walking. Forty-four participants with chronic hemiparesis walked at their self-selected speeds and eighteen nondisabled control subjects of similar ages walked at predetermined matched speeds while three-dimensional ground reaction forces and body-segment kinematics were recorded. Magnitude of paretic LL was reduced, while duration was increased compared with the nonparetic leg and nondisabled controls walking at matched speeds. The paretic LL and LU was significantly correlated with average leg angle, while the nonparetic leg significantly correlated with average knee angle. Three different patterns of LL and LU were identified (concave, convex, and linear). Individuals with hemiparesis make several biomechanical adjustments that minimize LL of the paretic leg. LL deviations were more pronounced with increased lateral placement of the paretic foot and with decreased functional gait speed. Characterization of these deviations may inspire new strategies for rehabilitation.
\end{abstract}

Key words: gait, hemiparesis, knee angle, leg angle, loading, magnitude, pattern, stroke, timing, unloading, vertical ground reaction forces.

\section{INTRODUCTION}

Hemiparesis following stroke results in poorly coordinated and asymmetric gait. In addition to the welldescribed kinematic asymmetries, there are also some less-described kinetic asymmetries. Kinetic parameters (e.g., joint moments, powers, and ground reaction forces [GRFs]) are particularly important because of their dependence on both kinematics and muscle activity and can provide insight into the underlying causes of changes in walking patterns. In particular, the vertical GRF has been used to characterize poststroke weight-bearing [1-2]. The paretic leg typically exhibits reduced weight-bearing compared to the nonparetic leg (i.e., neither limb loading (LL) nor limb unloading (LU) are symmetric) [3-8]. LL and LU asymmetry are important contributing factors to postural instability, reduced dynamic balance, increased postural sway, and thus falls [6]. Reduced paretic (as

\footnotetext{
Abbreviations: ANOVA $=$ analysis of variance, GRF = ground reaction force, $\mathrm{LL}=$ limb loading, $\mathrm{LU}=$ limb unloading, $\mathrm{VA}=$ Department of Veterans Affairs.

*Address all correspondence to Steven A. Kautz, PhD; Health Sciences and Research, 77 President Street, MSC 700, Charleston, SC 29425; 843-792-3867; fax: 843-7921358. Email: kautz@musc.edu

http://dx.doi.org/10.1682/JRRD.2011.02.0018
} 
compared with nonparetic) LL has been reported in static and quasi-static tasks such as standing in different stance positions [1,9-10], stepping on stairs of different heights [8], and sitting to standing from a chair [10] and has been related to walking ability poststroke [11-13]. However, studies typically report reduced paretic LL without quantifying the temporal characteristic of LL and LU, which may provide important information for understanding loading. While it is often assumed clinically that reduced loading of the paretic leg is indicative of poor walking and recovery, LL and LU asymmetry during walking in individuals with hemiparesis has been poorly quantified and the relationship with walking performance or stroke severity is unknown.

Walking involves acceleration of all individual body segments such that the whole body's center of mass is mostly positioned outside the base of support. Therefore, measuring LL and LU ability during static standing (standing on force plates and transferring weight from one leg to the other while the whole-body center of mass stays between the two feet) does not provide much detail about walking ability. In addition to forward and vertical acceleration of the whole-body center of mass, walking also involves mediolateral acceleration of the center of mass. Though the magnitudes of movement and forces in the mediolateral direction are small, they are expected to be important to facilitate LL and LU. This would be consistent with the observation that manual assistance provided at the pelvis by trainers during locomotor training to produce mediolateral movement of the body, presumably to facilitate appropriate weight shift, appears to improve walking patterns and increase stability [14].

Multiple paretic and nonparetic LL and LU patterns can be employed by individuals with hemiparesis to maintain steady-state walking. It is important to quantify these patterns to understand the differences in asymmetry between subjects during steady-state walking. The presence of multiple loading patterns would be consistent with the observation that subjects with poststroke hemiparesis exhibit multiple kinematic patterns to successfully maintain steady-state walking. The kinematic characteristics may provide more insight into the various patterns of LL and LU exhibited by different individuals. Therefore, we suggest that classifying poststroke individuals based on their LL and LU and evaluating the relationship of these parameters to kinematic and kinetic measures will provide deeper understanding of the effects of weight support and its influence on dynamic stability during walking.

The main purpose of this study was to determine the asymmetry in magnitude and duration of LL and LU in individuals with hemiparesis and its relationship with (1) functional walking status and (2) specific kinematic and kinetic variables during walking. We hypothesize that LL and LU in the paretic and nonparetic leg will differ with functional walking status and that the asymmetry will be related to knee angle and mediolateral leg angle (angle between vertical and the frontal plane projection of the line connecting the center of masses of the pelvis and foot). Since the magnitude of mediolateral forces are directly related to the mediolateral accelerations of the whole-body center of mass, we hypothesize that they will be intimately related to weight shift; thus, we will also establish the relationship between mediolateral forces and LL and LU. Detailed understanding of LL and LU asymmetry will provide additional insight into the changes in locomotion in poststroke individuals and potentially inspire new strategies to rehabilitate walking.

\section{METHODS}

\section{Participants}

Data were collected from 44 individuals with chronic hemiparesis (age $=60.2 \pm 12.3 \mathrm{yr}, 19$ females, 19 leftside hemiparesis) and 18 similarly aged nondisabled individuals (age $=66.2 \pm 10.0 \mathrm{yr}, 4$ males) at the Department of Veterans Affairs (VA)-University of Florida Human Motor Performance Laboratory in Gainesville, Florida. To be included in the study, individuals had to have hemiparesis secondary to a single-onset unilateral stroke, the ability to ambulate independently with or without an assistive device over $10 \mathrm{~m}$ on a level surface, and the ability to walk regularly in the home environment. Exclusion criteria were any orthopedic or neurologic conditions in addition to stroke that might limit hip and knee extension or ankle plantar flexion relative to neutral or inability to provide informed consent. Three categories of subjects with different stroke severity were identified based on functional walking status as defined by selfselected overground walking speeds [15]: individuals who walked $<0.4 \mathrm{~m} / \mathrm{s}$ (household ambulators), $0.4-0.8 \mathrm{~m} / \mathrm{s}$ (limited community ambulators), or $>0.8 \mathrm{~m} / \mathrm{s}$ (community ambulators). 


\section{Experimental Protocol}

Retroreflective markers were placed at specified bony prominences on the body. Clusters of reflective markers attached to rigid bodies were also placed firmly on the pelvis and each thigh, shank, and foot. All participants walked on an instrumented split-belt treadmill (Techmachine; Andrezieux-Boutheon, France) without using any assistive device or ankle foot orthosis for three walking trials at their self-selected treadmill walking speed. The treadmill was started at a substantially slower speed than subjects' overground walking speeds. The treadmill speed was then increased gradually until a comfortable walking speed for each subject was achieved. In order to ensure the safety of the ankle, subjects feeling unstable without an ankle foot orthosis were allowed to wear an ankle air cast (Aircast AirSport; Vista, California), which provided ankle stability in the mediolateral plane without limiting mobility in the sagittal plane. Data were collected for $30 \mathrm{~s}$ after the subject walked for $\sim 10 \mathrm{~s}$ to achieve steady state. Three-dimensional GRF and kinematic parameters for each leg were obtained as the subjects walked on the treadmill.

The subjects did not use any assistive device during data collection. However, a harness system mounted to the ceiling of the laboratory was used for safety in case participants lost their balance. The harness was worn across the shoulders and chest of participants, and no body weight was off-loaded by the system. A physical therapist stood near the subjects, but did not provide any assistance as they walked over the treadmill. Nondisabled control subjects walked at their self-selected speeds as well as at speeds of $0.3 \mathrm{~m} / \mathrm{s}$ and $0.6 \mathrm{~m} / \mathrm{s}$ to provide control data at speeds comparable to those of the hemiparetic individuals. Nondisabled control subjects practiced walking at each speed, and data collection did not begin until the subjects felt comfortable at the slow speeds.

A 12-camera motion analysis system (Vicon Motion Systems; Oxford, England) was used to collect the data as subjects walked on the instrumented treadmill. Customized MATLAB (The MathWorks, Inc; Natick, Massachusetts) programs were used for data analysis. Kinematic data were low-pass filtered using a fourthorder, zero-lag Butterworth filter with a $10 \mathrm{~Hz}$ cutoff frequency. GRFs were sampled at $2,000 \mathrm{~Hz}$ and low-pass filtered using a fourth-order, zero-lag Butterworth filter with a $20 \mathrm{~Hz}$ cutoff frequency. GRF data were normalized to each subject's body weight, and the gait cycle was expressed as percent of paretic leg gait cycle (i.e., paretic foot contact to paretic foot contact). LL and LU occur dur- ing the double-limb support phases of the gait cycle, and therefore, all variables were calculated in these two phases.

\section{Magnitude of Limb Loading and Unloading}

Average LL and LU were quantified by the average vertical GRF in the first and second double-support phases, respectively (Figure 1(a), Figure 2(a)). The legs in the first and second double-support phase were defined as loading and unloading, respectively (i.e., when one leg
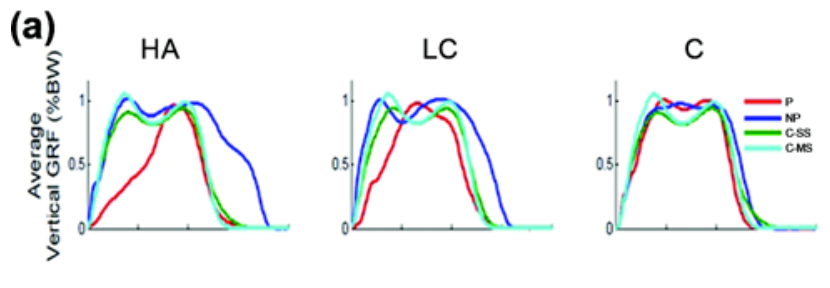

(b)
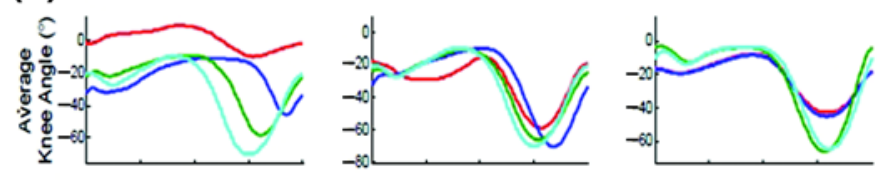

(c)
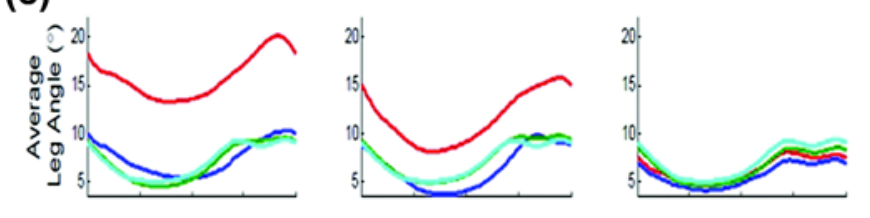

(d)
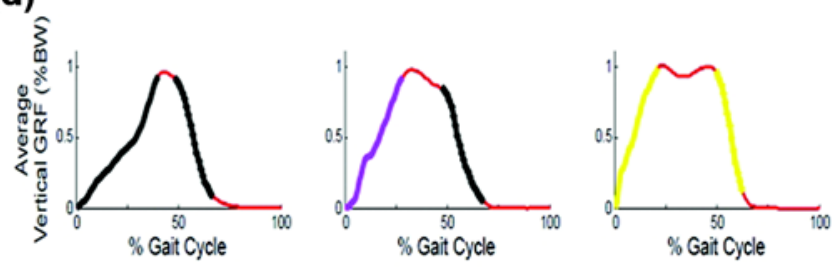

Figure 1.

(a) Limb loading and unloading. (b) Knee angle, (c) leg angle, and (d) loading and unloading pattern in one representative subject of each speed-based functional group (column $1=$ household ambulators [HAs]; column 2 = limited community ambulators [LCs]; and column $3=$ community ambulators [Cs]). Red is paretic leg (P), blue is nonparetic leg (NP), aqua blue is control at matched speeds (C-MS), and green is control at selfselected speeds (C-SS). Black highlight in (d) represents concave pattern; purple shows convex pattern and yellow is linear pattern. $\mathrm{BW}=$ body weight, GRF = ground reaction force. 

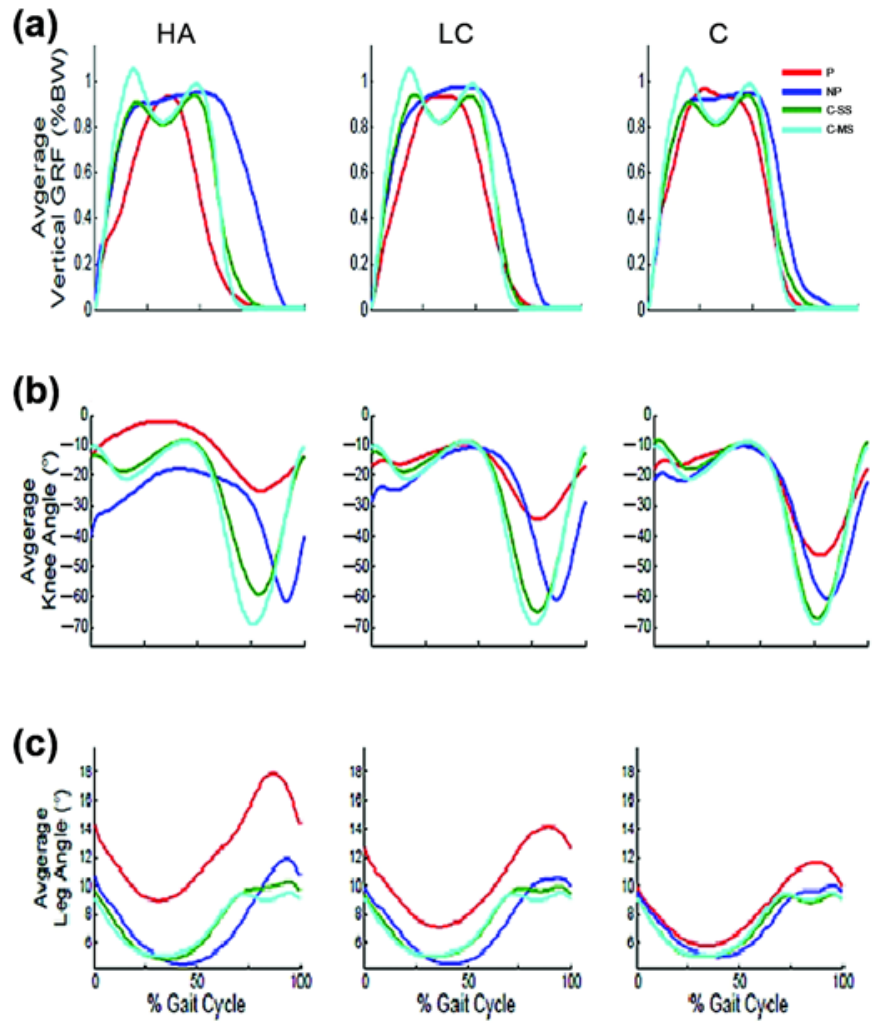

Figure 2.

(a) Limb loading and unloading. (b) Knee angle and (c) mediolateral leg angle of all individuals in each functional group (based on speed). First column represents household ambulators (HAs); second column limited community ambulators (LCS), while third column community ambulators (Cs). Red represents paretic leg $(P)$, blue nonparetic leg (NP), green control at selfselected speed (C-SS), aqua blue control at matched speeds (C-MS). BW = body weight, GRF = ground reaction force.

is being loaded the other is being unloaded in the same double-limb support phase).

\section{Timing of Limb Loading and Unloading}

The timing of LL was calculated for each leg as the crossover point of the two vertical GRF curves during the double-limb support phase (i.e., the percent gait cycle from the beginning of the double support until the majority of weight shifts from the leg in second double support to the leading leg in first double support) (Figure 3). Thus, the timing of LL of one leg is equal to that of LU in the other leg and expressed as a percentage of the double support phase (i.e., $50 \%=$ symmetric).

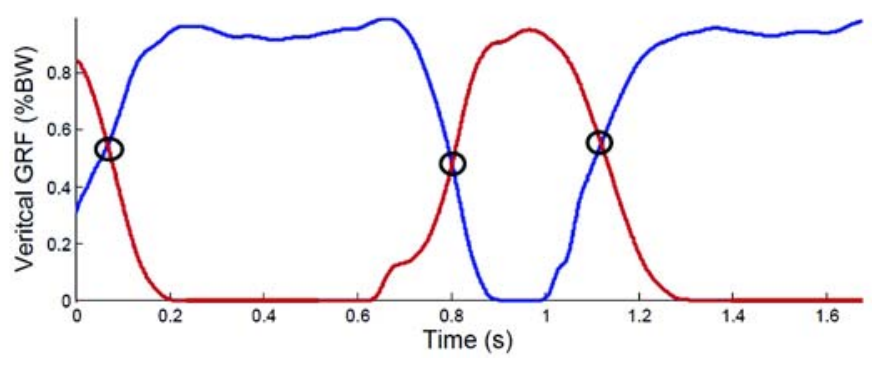

Figure 3.

Crossover point (circled in black) during paretic loading and unloading during walking. $\mathrm{BW}=$ body weight, $\mathrm{GRF}=$ ground reaction force.

\section{Pattern of Limb Loading and Unloading}

The pattern of LL and LU was calculated from the shape of the vertical GRF, from which we identified three patterns: concave curvature, convex curvature, and linear (Figure 1(d)). Concave curvature was associated with initially slow and then rapid loading of the limb. In contrast, convex curvature was associated with initially rapid and then slow loading of the limb. A linear shape of vertical GRF was indicative of uniform loading similar to the nondisabled control subjects. The vertical GRF was plotted separately from heel strike to the beginning of the single-limb support and from the end of the single-limb support to the toe-off highlighted in Figure 1(d). Thereafter, a best-fit line was plotted. The best-fit curve for a polynomial was calculated (JMP, SAS Institute, Inc; Cary North Carolina) for the vertical GRF values for the loading phase and unloading phase (end of single-leg support to toe-off) for each subject. Significance was set to $p<$ 0.001 as a correction for the large number of calculated curves. A significant first-order term meant a linear slope, which was a characteristic of the nondisabled control subjects, whereas a second-order coefficient term meant the LL and LU profile had a curvature that was a characteristic of LL and LU in individuals with hemiparesis. A positive second-order coefficient indicated concave curvature, while a negative coefficient indicated convex curvature.

\section{Kinematics}

The average leg angle in the mediolateral direction was calculated during each double-limb support phase as the angle between the vertical and the projection of the line connecting the center of mass of the pelvis with the center of mass of the foot in the frontal plane (Figure 1(c), 
Figure 2(c)). The average knee flexion-extension angle was calculated during each of the two double-limb support phases of the gait cycle (Figure 1(b), Figure 2(b)).

\section{Statistical Analysis}

For each of the measures of LL and LU, we ran separate one-way analyses of variance (ANOVAs) using SPSS, version 17 (IBM; Armonk, New York). One-way ANOVA was first used to compare the paretic and nonparetic leg of individuals with hemiparesis with nondisabled control subjects walking at matched and selfselected speeds. Another set of analyses using a one-way ANOVA was used to compare the LL and LU of individuals with hemiparesis classified into different speed groups with nondisabled control subjects walking at the corresponding matched speed and their self-selected speed. We also correlated LL and LU with the kinematic measures during each double-limb support phase of the gait cycle. Pearson correlation analysis was used to examine the relationship between LL and LU and various parameters selected (i.e., knee angle, leg angle, mediolateral GRF). The value of significance for all analysis was set at $\alpha=0.05$, and alpha values are reported.

\section{RESULTS}

The control subjects exhibited symmetric LL and LU patterns and kinematics between the two legs. Therefore, the average value of both legs for all parameters was used at all speeds.

\section{Magnitude of Limb Loading}

At the self-selected speeds, the average magnitude (normalized by body weight) of LL was less in the paretic leg than the nonparetic leg and similarly aged nondisabled control subjects. In contrast, at matched speeds, no significant difference existed between LL of the paretic leg and control subjects when considering all subjects with hemiparesis as a single group. When each speed group was considered separately, the reduced paretic leg LL was found to be related to the functional walking status of individuals with hemiparesis. The individuals walking at slower speeds had significant differences in LL compared with the control subjects, while community ambulators did not reveal any significant difference in LL (Table 1, Figure 1(a): individual representative subject, Figure 2(a): group average).

Table 1.

Comparisons of average of limb loading and unloading of paretic (P) and nonparetic (NP) leg of individuals walking at different speeds with nondisabled control subjects walking at matched speeds $\left(\mathrm{C}_{\mathrm{ms}}\right)$ and self-selected speed $\left(\mathrm{C}_{\mathrm{ss}}\right)$.

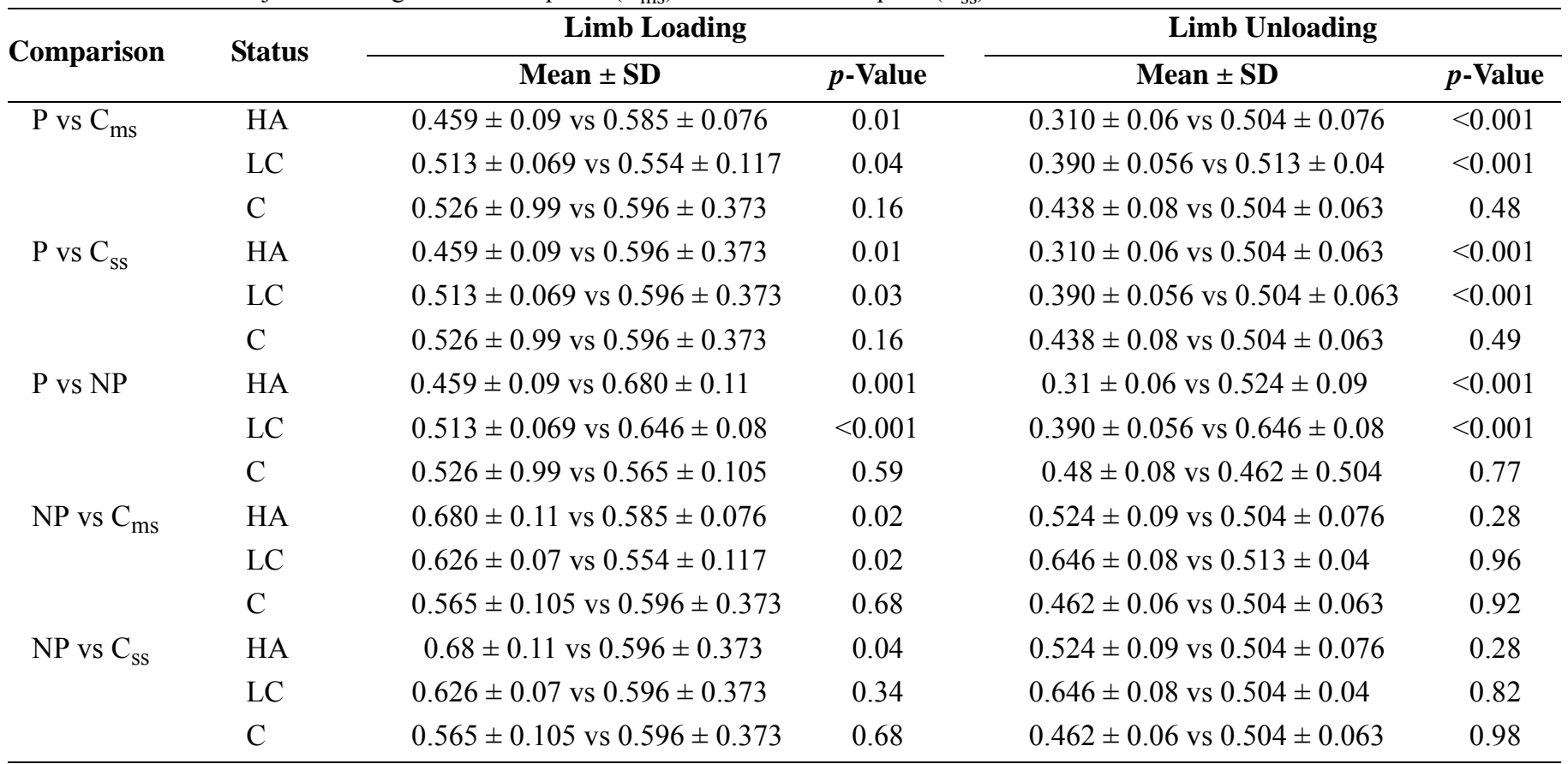




\section{Magnitude of Limb Unloading}

For household and limited community ambulators, the average magnitude of LU was significantly lower in the paretic leg than the nonparetic leg and similarly aged controls walking at self-selected and matched speeds. This lower average magnitude of force is indicative of greater unloading and was related to the severity of gait speed deficits. However, no significant difference was found in the LU profiles of the community ambulators when compared with nonparetic leg and control subjects walking (Table 1, Figure 1(a), Figure 2(a)).

\section{Timing and Pattern of Limb Loading and Unloading}

In addition to the difference in the magnitude of LL and LU, the timing of LL and LU of the paretic and nonparetic legs and nondisabled controls was significantly different. The transition of the majority of weight from the paretic to the nonparetic leg (timing of paretic LU) occurred at $32.0 \pm 8.2$ percent of the duration of the double-limb support phase, which was earlier than that from the nonparetic leg to the paretic leg (timing of nonparetic LU $51.6 \% \pm 9.9 \%)$ or control legs $(44.5 \% \pm 4.6 \%)$. This can be related to the information gained by the duration of the second double-limb support phase of the paretic $(28.0 \% \pm 4.2 \%$ of the gait cycle or $0.427 \mathrm{~s} \pm 0.07 \mathrm{~s})$ or nonparetic $(25.8 \% \pm 2.9 \%$ of the gait cycle or $0.38 \pm 0.09 \mathrm{~s})$ legs. This difference in timing of LL and LU was further investigated in each of the functional groups. One-way ANOVA analysis revealed that the average LL time of the nonparetic leg (transition of the weight from the paretic to nonparetic leg) in the household ambulators was significantly less than the limited community ambulators and the community ambulators. In addition, the LU time of the paretic leg of household ambulators was greater than limited community ambulators and community ambulators (Table 2 ).

In addition to the difference in timing of LL and LU, most subjects with hemiparesis were clustered into three groups based on the curvature of the LL and LU profiles of the paretic leg. Concave curvature of the LL profile was presented by 12 subjects: 10 household ambulators, 1 limited community ambulator, and 1 community ambulator. On the other hand, 20 subjects presented with convex curvature of the LL profile: 11 limited community ambulators, 4 household ambulators, and 5 community ambulators. Only 4 subjects presented with LL linear profile, 3 of whom were limited community ambulators and 1 of whom was a community ambulator. The side of
Table 2.

Average percentage of double-limb support phase required for transition of weight from paretic (P) to nonparetic (NP) (P to NP) and nonparetic to paretic (NP to $\mathrm{P})$. Data presented as mean \pm standard deviation.

\begin{tabular}{lll}
\hline Status & $\begin{array}{l}\text { LL of NP } \\
\text { (P to NP) }\end{array}$ & $\begin{array}{l}\text { LU of NP } \\
\text { (NP to P) }\end{array}$ \\
\hline HA & $22.5 \pm 8.9$ & $57.6 \pm 9.1$ \\
LC & $30.4 \pm 4.8$ & $50.1 \pm 8.6$ \\
C & $39.8 \pm 7.7$ & $47.1 \pm 6.9$
\end{tabular}

$\mathrm{C}=$ community ambulators $(>0.8 \mathrm{~m} / \mathrm{s}), \mathrm{HA}=$ household ambulators $(<0.4 \mathrm{~m} / \mathrm{s})$, $\mathrm{LC}=$ limited community ambulators $(0.4-0.8 \mathrm{~m} / \mathrm{s}), \mathrm{LL}=$ limb loading, $\mathrm{LU}=$ limb unloading.

paresis did not appear to be a factor in categorization of LL profiles. Eight subjects, which included six limitedcommunity ambulators and two community ambulators, could not be categorized because the GRF LL pattern was neither linear nor a second-order polynomial pattern (Table 3).

For LU, 31 subjects presented with concave curvature of the LU profile: 16 limited community ambulators, 11 household ambulators, and 4 community ambulators. In contrast, 7 subjects of all functional groups revealed convex curvature of the LU profile: 3 household ambulators, 2 limited community ambulators, and 2 community ambulators. Linear LU included only 2 community ambulators (Table 3). Four subjects could not be categorized because the GRF LU pattern was neither linear nor a second-order polynomial pattern (note that these subjects differed from the subjects mentioned in the pattern of LL analysis above). In summary, 8 out of 14 household ambulators had concave loading and 11 of 14 had concave unloading patterns. On the other hand, 11 out of $21 \mathrm{lim}-$ ited community ambulators had a convex loading pattern and 16 of 21 had a concave unloading pattern. The community ambulators did not show any consistent patterns.

\section{Relationship Between Mediolateral Ground Reaction Forces and Limb Loading and Unloading}

Mediolateral forces were not significantly correlated with the vertical GRF during both double-limb support phases of the paretic leg for first $(p=0.91)$ and second $(p=$ 0.34 ) double-limb support phases.

\section{Relationship Between Knee Angle and Limb Loading and Unloading}

There was no statistically significant correlation between average LL and LU and knee angle on the 
Table 3.

Loading and unloading details of hemiparetic subjects arranged based on their self-selected speed (household ambulators [HA] $<0.0 .4 \mathrm{~m} / \mathrm{s}$; limited community ambulators [LC] $0.4-0.8 \mathrm{~m} / \mathrm{s}$, and community ambulators [C] $>0.8 \mathrm{~m} / \mathrm{s}$ ).

\begin{tabular}{|c|c|c|c|c|c|}
\hline Status & $\begin{array}{c}\text { Speed } \\
(\mathrm{m} / \mathrm{s})\end{array}$ & $\begin{array}{c}\text { Paretic } \\
\text { Side }\end{array}$ & $\begin{array}{c}\text { Time } \\
\text { Since Stroke } \\
\text { (mo) }\end{array}$ & $\begin{array}{c}\text { LL } \\
\text { Pattern }\end{array}$ & $\begin{array}{c}\text { LU } \\
\text { Pattern }\end{array}$ \\
\hline \multirow[t]{14}{*}{ HA } & 0.35 & Right & 95 & Concave & Concave \\
\hline & 0.18 & Right & 62 & Concave & Concave \\
\hline & 0.26 & Right & 11 & Concave & Convex \\
\hline & 0.36 & Right & 31 & Concave & Convex \\
\hline & 0.15 & Right & 115 & Concave & Concave \\
\hline & 0.24 & Right & 29 & Concave & Concave \\
\hline & 0.35 & Right & 127 & Convex & Concave \\
\hline & 0.34 & Left & 94 & Convex & Concave \\
\hline & 0.39 & Left & 26 & Convex & Concave \\
\hline & 0.19 & Left & 26 & Convex & Concave \\
\hline & 0.21 & Left & 54 & Concave & Concave \\
\hline & 0.21 & Left & 9 & Concave & Concave \\
\hline & 0.35 & Left & 55 & Concave & Concave \\
\hline & 0.22 & Left & 114 & Concave & Convex \\
\hline \multirow[t]{21}{*}{$\mathrm{LC}$} & 0.70 & Left & 65 & Convex & Concave \\
\hline & 0.45 & Left & 12 & Convex & Concave \\
\hline & 0.63 & Left & 37 & Convex & Concave \\
\hline & 0.73 & Left & 62 & Convex & Concave \\
\hline & 0.75 & Left & 9 & Convex & Concave \\
\hline & 0.42 & Left & 10 & Concave & Convex \\
\hline & 0.51 & Left & 76 & Linear & Concave \\
\hline & 0.42 & Right & 58 & Convex & Concave \\
\hline & 0.63 & Right & 90 & Convex & Concave \\
\hline & 0.65 & Right & 67 & Convex & Convex \\
\hline & 0.62 & Right & 86 & Convex & Concave \\
\hline & 0.43 & Right & 411 & Linear & Concave \\
\hline & 0.40 & Right & 100 & $*$ & Concave \\
\hline & 0.48 & Right & 43 & $*$ & Concave \\
\hline & 0.44 & Left & 7 & $*$ & Concave \\
\hline & 0.40 & Left & 25 & $*$ & Concave \\
\hline & 0.80 & Left & 64 & $*$ & Concave \\
\hline & 0.42 & Left & 16 & $*$ & Concave \\
\hline & 0.46 & Left & 36 & Convex & * \\
\hline & 0.57 & Left & 11 & Convex & $*$ \\
\hline & 0.49 & Left & 95 & Linear & $*$ \\
\hline \multirow[t]{9}{*}{$\mathrm{C}$} & 1.05 & Left & 44 & Convex & Concave \\
\hline & 0.87 & Left & 15 & Convex & Concave \\
\hline & 0.90 & Left & 208 & Concave & Linear \\
\hline & 0.86 & Left & 116 & Linear & Linear \\
\hline & 0.84 & Right & 26 & Convex & Concave \\
\hline & 1.00 & Right & 153 & Convex & Convex \\
\hline & 0.99 & Right & 27 & Convex & $*$ \\
\hline & 0.80 & Right & 8 & $*$ & Convex \\
\hline & 0.99 & Left & 41 & $*$ & Concave \\
\hline
\end{tabular}

paretic leg (Figure 4(a)). However, there was a trend toward significance for LU, suggesting that individuals with greater unloading (lower average vertical GRF values) may have greater knee flexion $(r=-0.150, p=0.16$ for LL and knee angle; and $r=-0.213, p=0.05$ for LU and knee angle) (Figure 4(b)). Nonparetic leg LL was significantly related to knee angle $(r=-0.459, p=0.001)$ (Figure 4(c)), highlighting that the individuals with greater LL have less knee flexion, but the LU showed no significant correlation $(r=0.196, p=0.26)$ (Figure 4(d)).

\section{Relationship Between Mediolateral Leg Angle and Limb Loading and Unloading}

Subjects with reduced paretic leg LL placed the paretic leg further lateral relative to the center of mass of the pelvis. LL was negatively associated with the leg angle of the paretic side in the mediolateral direction during the first double-support phase of the gait cycle ( $r=$ $-0.451, p=0.002$ ) (Figure 5). The paretic leg mediolateral angle $\left(9.4^{\circ} \pm 2.6^{\circ}\right)$ was always significantly $(p<$ $0.05)$ greater than that of the nonparetic leg $\left(7.3^{\circ} \pm 2.0^{\circ}\right)$ and nondisabled controls walking at matched speeds (Figure 1(c), Figure 2(c)), further supporting that the paretic leg was placed more lateral from the center of mass of the body than the nonparetic leg and controls.

\section{DISCUSSION}

The paretic leg was loaded less (reduced magnitude) and more slowly (prolonged duration) and was unloaded more quickly than the nonparetic leg and nondisabled controls. These loading and unloading deviations were significantly correlated with lateral placement of the paretic foot relative to the pelvis and also functional walking status. Existing evidence in the literature has indicated asymmetry in the loading abilities of individuals with hemiparesis during static and quasi-static tasks $[1,9,16]$. In contrast to the paretic leg, and given the mechanical coupling between legs, the nonparetic leg was loaded more (increased magnitude) and more quickly (shortened duration) and was unloaded more slowly than the paretic leg and nondisabled controls. Also, these loading and unloading deviations were significantly correlated with nonparetic knee angle such that the increased loading was associated with increased knee extension. Furthermore, the reduced LL of the paretic leg and the increased compensatory LL of the nonparetic leg varied with the 
(a)

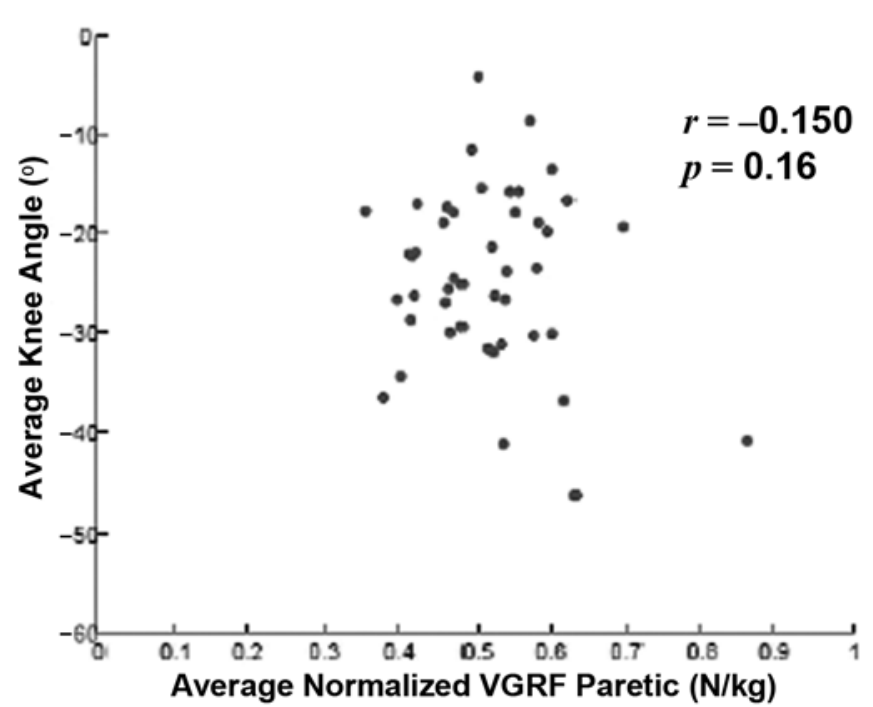

(c)

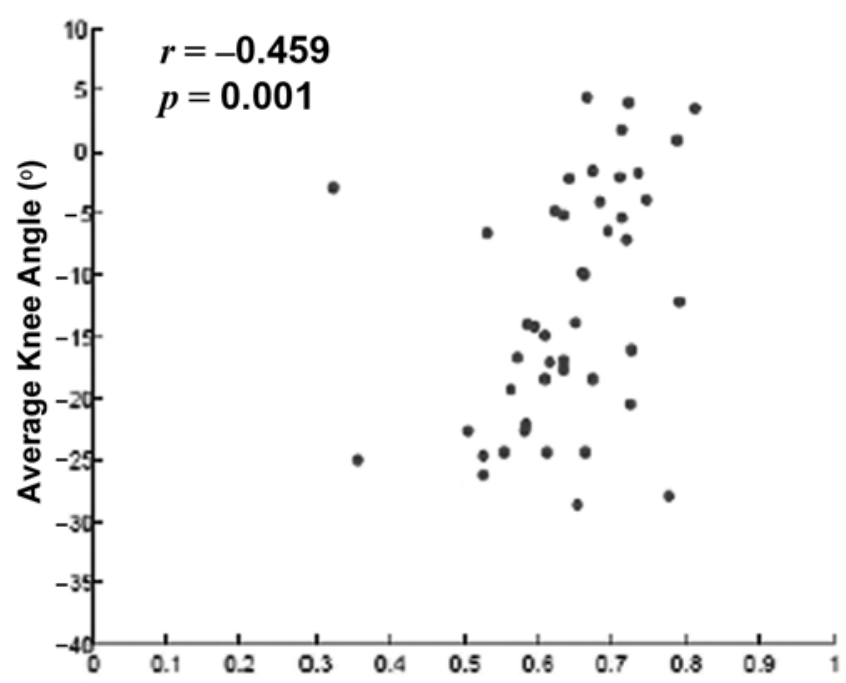

Average Normalized VGRF Nonparetic (N/kg) (b)

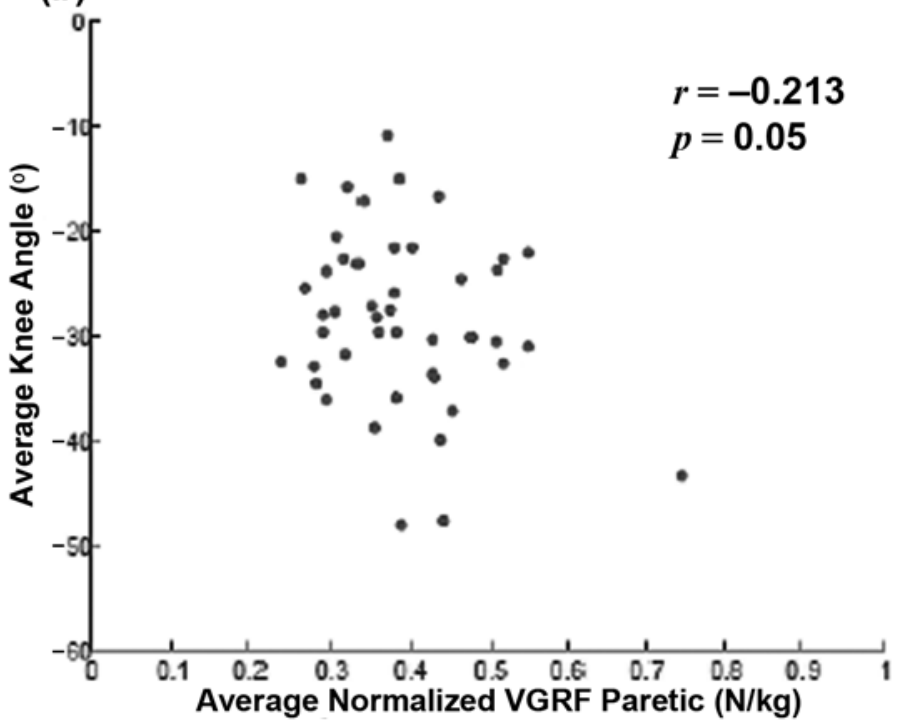

(d)

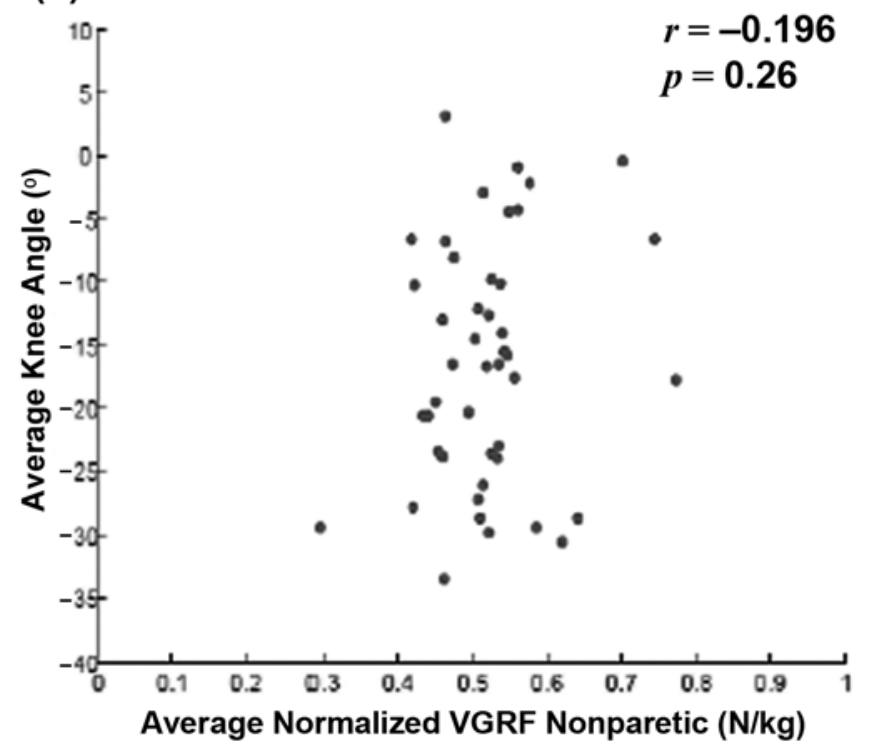

Figure 4.

Correlations between loading and unloading and average knee angle. (a) Paretic loading and knee angle, (b) paretic unloading and knee angle, (c) nonparetic loading and knee angle, (d) nonparetic unloading and knee angle. $x$-axis represents average magnitude of vertical ground reaction force (VGRF) (Newtons/kilogram); $y$-axis represents average knee angle (degrees).

severity of stroke (as defined by the self-selected walking speed). This is supported by significantly greater loading of the nonparetic leg (i.e., greater paretic unloading) during initial double-limb support in the household ambulators than the limited community and community ambulators. This supports the first part of our hypothesis that the LL and LU in the paretic leg will differ from the nonparetic leg and nondisabled control subjects in individuals with different functional walking status.

The compensation offered by the nonparetic leg can be explained by the phenomenon of "impairment and compensation" [17]. This phenomenon suggests that the greater the impairment of the paretic leg, the greater the compensation offered by the nonparetic leg to maintain 


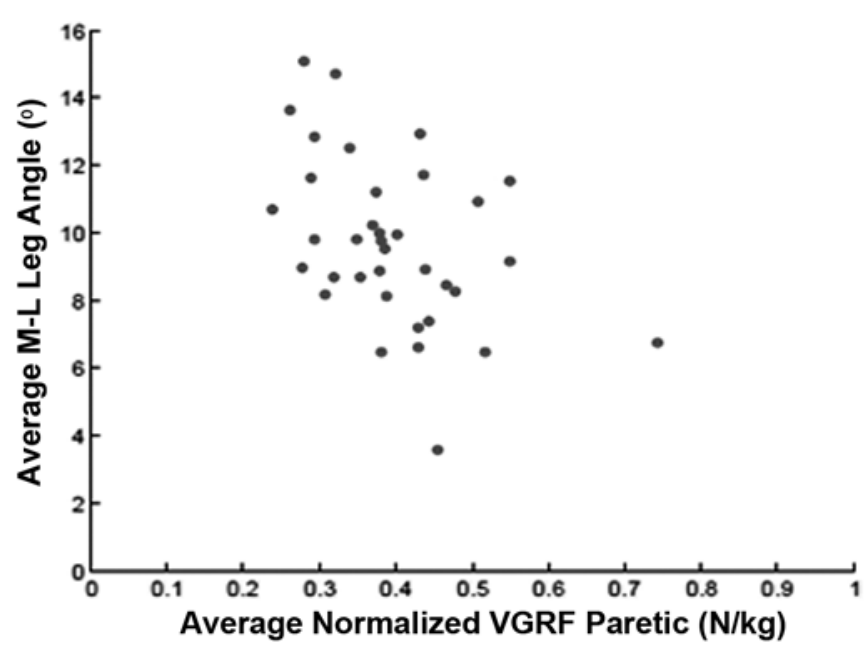

Figure 5.

Correlations between loading and average paretic mediolateral $(\mathrm{M}-\mathrm{L})$ leg angle. $x$-axis represents average magnitude of vertical ground reaction force (VGRF) (Newtons/kilogram); $y$-axis represents average $\mathrm{M}-\mathrm{L}$ leg angle (degrees).

stability during the ongoing task of walking. The asymmetry between the paretic and nonparetic leg in both LL and LU could be attributed to the weakness and decreased control of the paretic leg $[1,18]$. Physiologically, reduced loading of the paretic leg might be associated with reduced muscle activation during walking, which might result in increased impairments such as poor body support or propulsion. The LU is increased on the paretic side, meaning that the individuals with hemiparesis unload their paretic leg rapidly.

In addition to the asymmetry in the magnitude of LL and LU, it was important to study the timing of LL and LU because walking is a cyclic task produced as a result of synchronized phasic stimuli (hip extension and LL and LU). Individuals with hemiparesis walking at their selfselected speeds accept body weight much more slowly during the initial double-limb support of the paretic leg (than the nonparetic leg), whereas during terminal double-limb support, the weight is rapidly transferred to the nonparetic leg. This suggests a reluctance to load the paretic leg, resulting in reduced single-leg stance phase on the paretic leg and longer double-limb support phase.

Although the timing of LL and LU provides details about LL and LU asymmetry, it does not capture the variability of biomechanical adjustments made by individuals with hemiparesis as does the convex, concave, or linear shape of the LL and LU curves. Individuals with concave LL patterns were primarily household ambulators (i.e., walking at $<0.4 \mathrm{~m} / \mathrm{s}$ ), and a majority of the individuals with convex LL patterns were limited community ambulators (i.e., $0.4-0.8 \mathrm{~m} / \mathrm{s}$ ). The linear pattern comprised both limited community and community ambulating subjects (Figure 6). For the 10 subjects who displayed both concave LL and LU, the loading of both paretic and nonparetic legs was slow, suggesting that they spent a large proportion of the gait cycle in the doublelimb support phase and displayed slow loading of both the paretic and nonparetic leg. While we limited our loading and unloading phase analysis to the double-support regions, Figure 1(d) and Figure 2(a) show that loading and unloading was also occurring in the single-limb support phase and changing systematically across the different types of ambulators (note the progressively wider peaks in vertical GRF as functional walking status improves). Similar to the shape of the LL and LU curves, the shape
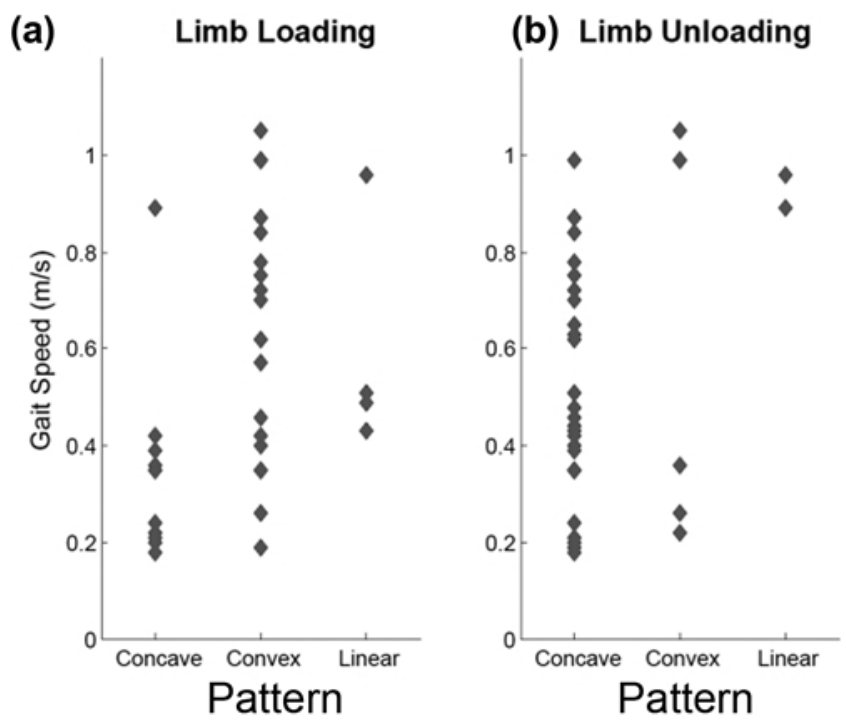

Figure 6.

Individuals with different (a) loading and (b) unloading patterns against their self-selected walking speed. Self-selected walking speed is used in this study to categorize individuals into three groups $(<0.4 \mathrm{~m} / \mathrm{s}=$ household ambulators, $0.4-0.8 \mathrm{~m} / \mathrm{s}=$ limited community ambulators, and $>0.8 \mathrm{~m} / \mathrm{s}=$ community ambulators). Each point on scatter plot represents hemiparetic individual. $x$ axis represents different loading and unloading patterns. Note: number of points looks less than total number of subjects because subjects with similar speeds are all represented with single point. 
of the peak in the vertical GRF may also be related to ambulatory status and additional biomechanical variables.

As previously mentioned, individuals with hemiparesis make several biomechanical adjustments to minimize loading of the paretic leg (i.e., by reducing LL and increasing LU, time to load, and knee flexion angle). Knee angle is an important component of body support during weight acceptance in order to prevent collapse and has been the most often reported kinematic variable in relation to walking ability poststroke [19-20]. The second part of our hypothesis was only partially supported, because knee angle was not significantly related to LL and LU of the paretic leg, but the trend toward significance suggested possible importance of knee angle in understanding LU. However, a significant negative relationship was reported on the nonparetic side. This highlights that individuals with greater loading have lesser knee flexion, suggesting that the nonparetic leg is maintained in extension to support body weight during the loading phase. Furthermore, we found that the knee was more flexed in the nonparetic leg than the paretic leg and nondisabled controls during both LL and LU. In addition, the difference in terminal double-limb support could be explained by reduced ability to plantar flex the ankle to create push-off power during the second double-limb support phase, and also the increased knee flexion could be credited to increased power generation requirements of the nonparetic leg (at hip, knee, and ankle) to compensate for the decreased power output from the paretic leg [21].

Additionally, LL and LU in subjects with hemiparesis is influenced by mediolateral leg angle. The results of our study support the observation that the hemiparetic individuals place their nonparetic leg close to their body while placing their paretic leg farther away [22], indicating that the mediolateral leg angle is greater on the paretic leg and significantly less on the nonparetic leg, thus validating the third component of our hypothesis. The lateral placement of the paretic leg may be a strategy to increase the base of support while walking or to minimize loading of the paretic leg. Minimizing loading of the paretic leg may be attributed to weakness of the paretic leg, or impairment following stroke, or fear of falling. Finally, we believe that the absence of a significant correlation between the mediolateral GRF and LL and LU may be due to high variability in the mediolateral forces from the compensatory gait pattern employed by individuals walking after stroke. Thus, this information might assist in design of better rehabilitation techniques.
For example, greater emphasis could be placed on the weight-shifting abilities of the household ambulators to promote faster and more physiologic walking speeds.

\section{NONNEUROLOGICAL IMPLICATIONS OF INCREASED LOADING OF NONPARETIC LEG}

Several researchers have recognized that stroke survivors experience excess bone loss in the paretic and lower limbs because of reduced physical activity [2324]. Bone loss in the lower limb is a particularly serious concern, as stroke survivors have a hip fracture risk two to four times higher than that of age- and sex-matched nondisabled individuals [25-26]. Furthermore, Worthen et al. [27] demonstrated the strongest correlations between bone density indexes based on the mean vertical GRF experienced during walking. They further suggested that these results have implications for the use of gait training as an osteoporosis countermeasure. In particular, the role of increased vertical GRFs in bone mineral density maintenance is promising as a therapeutic intervention.

In addition to individuals with hemiparesis, there is evidence in individuals postamputation that suggests osteoarthritis of the joints in the intact leg (e.g., knee [28] and hip [29] joints) due to changes in the direction of the GRFs and greater leg loading [30]. In addition, subjects with poliomyelitis typically have asymmetrical disease, with greater forces transmitted across the unaffected leg resulting in greater symptoms on the unaffected leg than the affected leg [31]. Thus, greater loading of the nonparetic leg might result in musculoskeletal changes over time that might further interfere with the walking ability of those with hemiparesis. Therefore, a better understanding of LL and LU deviations during gait may allow for more objectively directed therapy to address the abnormalities of both the paretic and nonparetic limbs to promote better functional gait and minimize long-term musculoskeletal changes.

\section{CONCLUSIONS}

The results of this study indicate that the magnitude of paretic LL was reduced, while duration was increased compared with the nonparetic leg and nondisabled controls walking at matched speeds. In addition, three different patterns of LL and LU were also identified (concave, 
convex, and linear). The paretic LL and LU were significantly correlated with average leg angle, while the nonparetic leg significantly correlated with average knee angle, indicating that individuals with hemiparesis poststroke make several biomechanical adjustments to maintain a steady walking state. LL deviations were more pronounced with increased lateral placement of the paretic foot and with decreased functional gait speed. Characterization of these deviations may inspire new strategies for rehabilitation.

\section{ACKNOWLEDGMENTS}

\author{
Author Contributions: \\ Study concept and design: S. A. Kautz, B. Raja. \\ Acquisition of data: B. Raja. \\ Analysis and interpretation of data: S. A. Kautz, B. Raja. \\ Drafting of manuscript: B. Raja. \\ Critical revision of manuscript for important intellectual content: \\ S. A. Kautz, R. R. Neptune. \\ Statistical analysis: B. Raja. \\ Obtained funding: S. A. Kautz, R. R. Neptune. \\ Study supervision: S. A. Kautz.
}

Financial Disclosures: The authors have declared that no competing interests exist.

Funding/Support: This material was based on work supported by the National Institutes of Health (grant RO1 HD46820) and the VA Rehabilitation Research and Development Service.

Institutional Review: All participants in the study signed a written informed consent, and the study was approved by the Institutional Review Board at the University of Florida.

Participant Follow-Up: The authors do not plan to inform participants of the publication of this study. However, participants were informed that the work was intended to be published at the time of consent.

Additional Contributions: The authors would like to thank Erin Carr, Helen Emery, Dr. Mark Bowden, Kelly Rooney, and Ryan Knight for help with the data collection and processing and Ryan Knight for help with generating the figures.

Disclaimer: The contents of this article are solely the responsibility of the authors and do not necessarily represent the official views of the National Institutes of Health, National Institute of Child Health and Human Development, or VA.

\section{REFERENCES}

1. Pai YC, Rogers MW, Hedman LD, Hanke TA. Alterations in weight-transfer capabilities in adults with hemiparesis. Phys Ther. 1994;74(7):647-57, discussion 657-59. [PMID:8016197]
2. Bohannon RW, Larkin PA. Lower extremity weight bearing under various standing conditions in independently ambulatory patients with hemiparesis. Phys Ther. 1985;65(9): 1323-25. [PMID:4034666]

3. Shumway-Cook A, Anson D, Haller S. Postural sway biofeedback: its effect on reestablishing stance stability in hemiplegic patients. Arch Phys Med Rehabil. 1988;69(6): 395-400. [PMID:3377664]

4. Winstein CJ, Gardner ER, McNeal DR, Barto PS, Nicholson DE. Standing balance training: effect on balance and locomotion in hemiparetic adults. Arch Phys Med Rehabil. 1989;70(10):755-62. [PMID:2802955]

5. Mizrahi J, Solzi P, Ring H, Nisell R. Postural stability in stroke patients: vectorial expression of asymmetry, sway activity and relative sequence of reactive forces. Med Biol Eng Comput. 1989;27(2):181-90. [PMID:2601436] http://dx.doi.org/10.1007/BF02446228

6. Sackley CM. Falls, sway, and symmetry of weight-bearing after stroke. Int Disabil Stud. 1991;13(1):1-4.

[PMID:1917796]

http://dx.doi.org/10.3109/03790799109166267

7. Rode G, Tiliket C, Boisson D. Predominance of postural imbalance in left hemiparetic patients. Scand J Rehabil Med. 1997;29(1):11-16. [PMID:9084100]

8. Laufer Y, Dickstein R, Resnik S, Marcovitz E. Weightbearing shifts of hemiparetic and nondisabled adults upon stepping on stairs of various heights. Clin Rehabil. 2000; 14(2):125-29. [PMID:10763788] http://dx.doi.org/10.1191/026921500674231381

9. Goldie PA, Matyas TA, Evans OM, Galea M, Bach TM. Maximum voluntary weight-bearing by the affected and unaffected legs in standing following stroke. Clin Biomech (Bristol, Avon). 1996;11(6):333-42. [PMID:11415642] http://dx.doi.org/10.1016/0268-0033(96)00014-9

10. Eng JJ, Chu KS. Reliability and comparison of weightbearing ability during standing tasks for individuals with chronic stroke. Arch Phys Med Rehabil. 2002;83(8):1138-44. [PMID:12161837] http://dx.doi.org/10.1053/apmr.2002.33644

11. Carlsöö S, Dahlöf AG, Holm J. Kinetic analysis of the gait in patients with hemiparesis and in patients with intermittent claudication. Scand J Rehabil Med. 1974;6(4):166-79. [PMID:4458059]

12. Bogarth E, Richards C. Gait analysis and re-learning of gait control in hemiplegic patients. Physiother Can. 1981;33: 223-30.

13. Wall JC, Turnbull GI. Gait asymmetries in residual hemiplegia. Arch Phys Med Rehabil. 1986;67(8):550-53. [PMID:3741082]

14. Behrman AL, Harkema SJ. Locomotor training after human spinal cord injury: a series of case studies. Phys Ther. 2000;80(7):688-700. [PMID:10869131] 
15. Perry J, Garrett M, Gronley JK, Mulroy SJ. Classification of walking handicap in the stroke population. Stroke. 1995; 26(6):982-89. [PMID:7762050] http://dx.doi.org/10.1161/01.STR.26.6.982

16. de Haart M, Geurts AC, Dault MC, Nienhuis B, Duysens J. Restoration of weight-shifting capacity in patients with postacute stroke: a rehabilitation cohort study. Arch Phys Med Rehabil. 2005;86(4):755-62. [PMID:15827928] http://dx.doi.org/10.1016/j.apmr.2004.10.010

17. Olney SJ, Richards C. Hemiparetic gait following stroke. Part I: Characteristics. Gait Posture. 1996;4:136-48. http://dx.doi.org/10.1016/0966-6362(96)01063-6

18. van Asseldonk EH, Buurke JH, Bloem BR, Renzenbrink GJ, Nene AV, van der Helm FC, van der Kooij H. Disentangling the contribution of the paretic and nonparetic ankle to balance control in stroke patients. Exp Neurol. 2006; 201(2):441-51. [PMID:16814283] http://dx.doi.org/10.1016/j.expneurol.2006.04.036

19. Mulroy S, Gronley J, Weiss W, Newsam C, Perry J. Use of cluster analysis for gait pattern classification of patients in the early and late recovery phases following stroke. Gait Posture. 2003;18(1):114-25. [PMID:12855307] http://dx.doi.org/10.1016/S0966-6362(02)00165-0

20. De Quervain IA, Simon SR, Leurgans S, Pease WS, McAllister D. Gait pattern in the early recovery period after stroke. J Bone Joint Surg Am. 1996;78(10):1506-14. [PMID:8876578]

21. Chen G, Patten C, Kothari DH, Zajac FE. Gait differences between individuals with post-stroke hemiparesis and nondisabled controls at matched speeds. Gait Posture. 2005; 22(1):51-56. [PMID:15996592] http://dx.doi.org/10.1016/j.gaitpost.2004.06.009

22. Balasubramanian CK, Neptune RR, Kautz SA. Foot placement in a body reference frame during walking and its relationship to hemiparetic walking performance. Clin Biomech (Bristol, Avon). 2010;25(5):483-90. [PMID:20193972] http://dx.doi.org/10.1016/j.clinbiomech.2010.02.003

23. Prince RL, Price RI, Ho S. Forearm bone loss in hemiplegia: a model for the study of immobilization osteoporosis. J Bone Miner Res. 1988;3(3):305-10. [PMID:3213623] http://dx.doi.org/10.1002/jbmr.5650030309

24. Mulley G, Espley AJ. Hip fracture after hemiplegia. Postgrad Med J. 1979;55(642):264-65. [PMID:471862] http://dx.doi.org/10.1136/pgmj.55.642.264

25. Kanis J, Oden A, Johnell O. Acute and long-term increase in fracture risk after hospitalization for stroke. Stroke.
2001;32(3):702-6. [PMID:11239190]

http://dx.doi.org/10.1161/01.STR.32.3.702

26. Ramnemark A, Nyberg L, Borssén B, Olsson T, Gustafson Y. Fractures after stroke. Osteoporos Int. 1998;8(1):92-95. [PMID:9692083] http://dx.doi.org/10.1007/s001980050053

27. Worthen LC, Kim CM, Kautz SA, Lew HL, Kiratli BJ, Beaupre GS. Key characteristics of walking correlate with bone density in individuals with chronic stroke. J Rehabil Res Dev. 2005;42(6):761-68. [PMID:16680613] http://dx.doi.org/10.1682/JRRD.2005.02.0036

28. Burke MJ, Roman V, Wright V. Bone and joint changes in lower limb amputees. Ann Rheum Dis. 1978;37(3):252-54. [PMID:150823] http://dx.doi.org/10.1136/ard.37.3.252

29. Kulkarni J, Adams J, Thomas E, Silman A. Association between amputation, arthritis and osteopenia in British male war veterans with major lower limb amputations. Clin Rehabil. 1998;12(4):348-53. [PMID:9744670] http://dx.doi.org/10.1191/026921598672367610

30. Robbins S, Waked E, Krouglicof N. Vertical impact increase in middle age may explain idiopathic weight-bearing joint osteoarthritis. Arch Phys Med Rehabil. 2001; 82(12):1673-77. [PMID:11733881] http://dx.doi.org/10.1053/apmr.2001.26255

31. Harrington IJ. Knee joint forces in normal and pathological gait. In: Niwa S, Perren SM, Hatton T, editors. Biomechanics in orthopaedics. Tokyo (Japan): Springer-Verlag; 1992.

Submitted for publication February 11, 2011. Accepted in revised form March 21, 2012.

This article and any supplemental material should be cited as follows:

Raja B, Neptune RR, Kautz SA. Quantifiable patterns of limb loading and unloading during hemiparetic gait: Relation to kinetic and kinematic parameters. J Rehabil Res Dev. 2012;49(9):1293-1304.

http://dx.doi.org/10.1682/JRRD.2011.02.0018

ResearcherID: Steven A. Kautz, PhD: I-4164-2012

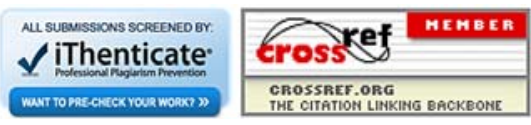

